\section{JUBILEE OF THE DISCOVERY OF THE ELECTRON}

$\mathrm{I}^{\mathrm{N}}$ 1897, Prof. J. J. Thomson, working in the Cavendish Laboratory at Cambridge, discovered that cathode rays consist of negatively charged particles (electrons, as they afterwards came to be known) many times smaller in size than the smallest atoms known. During the fifty years that have elapsed since that discovery, it has been established that electrons form an essential part of all matter. Numerous practical applications of the use of electrons have been found, and vast new industries have been set up based on electronic devices. On the theoretical side, the discovery of the electron has opened the way to research into the structure of the atom, and, more recently, into that of the nucleus, leading finally, for better or worse, to the release of atomic energy.

To mark the jubilee of the discovery of the electron, celebrations were held recently in London by the Institute of Physics and the Physical Society in collaboration with the Institution of Electrical Engineers. On the afternoon of September 25, at the Royal Institution, Prof. J. A. Crowther gave a lecture on the history and early development of the discovery. He was followed by Prof. R. E. Peierls, who spoke on electrons in modern theoretical physics. In the evening, a public lecture was delivered to a very large non-technical audience at the Central Hall, Westminster, by Sir Clifford Paterson, director of the Research Laboratories of the General Electric Co., Ltd., on "The Electron Liberated". On the following day, the Electron Jubilee Exhibition at the Science Museum, South Kensington (see Nature, Oct. 4, p. 461), was formally opened. At the Institution of Electrical Engineers, a posthumous portrait of $\operatorname{Sir}$ J. J. Thomson, painted by Mr. Francis Hodge, was unveiled by 'J. J.'s' son, Sir George Thomson, who then addressed the assembly on the electron in research. $\mathrm{He}$ was followed by Dr. T. E. Allibone, who spoke on electrons in industry.

Prof. A. M. Tyndall, president of the Institute of Physies, in introducing Prof. J. A. Crowther, stated that the suggestion to celebrate the jubilee of the discovery of the electron had originated from a remark made by Dr. A. J. Maddock. The suggestion was taken up enthusiastically by the Board of the Institute of Physics, which then invited the Physical Society and the Institution of Electrical Engineers to join in the organisation of a series of meetings which would be thoroughly representative of the two sciences-physics and electrical engineeringwhich had been most directly affected by the discovery of the electron. Prof. Tyndall invited the meeting to send the following message of greeting to Lady Thomson, who was prevented from being at the meeting. "Representatives of science and industry, together with old friends, assembled at the Royal Institution to-day to commemorate the fiftieth anniversary of the discovery of the electron by your husband, send you warm greetings and best wishes."

Opening his lecture, Prof. Crowther said that in order to appreciate to the full the discovery of the electron and the genius of its discoverer, Sir J. J. Thomson, it was necessary to be aware of the ideas prevalent in the pre-electron era, and perhaps also the facilities available then. He therefore briefly outlined the position of physical knowledge in 1897 and the course of events which led up to Thomson's experiments. Prior to 1890 , the study of electrical discharges in a vacuum tube had progressed very slowly, no doubt partly due to the extremely laborious methods of evacuation which had to be used, and also partly to the lack of serious examination of the beautiful phenomena observed. Schuster, in 1890 , made the first real attack on the problem of elucidating the nature of the cathode rays, when he took the trouble to measure the magnetic deflexion of the rays in a measured magnetic field. The accepted view of British physicists at the time was that the cathode ray particles were atoms, so that the energy given to them by the electrical field would be all used up in driving the particles through the residual gas present in the discharge tube. Working on this assumption, Schuster obtained a value of 2,000 for the ratio of the charge $e$ to the mass $m$ of the cathode particle, a value which is consistent with the cathode particle being a nitrogen atom. The experiments performed by Hertz, Lenard and Perrin during the next few years did not help to clarify the situation, but provided rather conflicting evidence.

This was the baffling state of affairs when J. J. Thomson entered the lecture room of the Royal Institution on the evening of Friday, April 30, 1897, and delivered his now historic lecture on "Cathode Rays". Quoting from Thomson's lecture, Prof. Crowther showed how the results of Perrin's and Lenard's experiments were first carefully analysed by Thomson, leading him to the conclusion that "the size of the carriers must be small compared with the dimensions of ordinary atoms and molecules"; and then, further, how Thomson startled his audience with the bold and revolutionary conception that the atom has a structure which could be explored. By the October of 1897, Thomson was in a position to present, in the Philosophical Magazine, additional experimental evidence in support of his claims. This evidence included the now famous and elegant measurement of $\mathrm{e} / \mathrm{m}$ for cathode particles. Finally, at the British Association meeting in 1899, Thomson announced that he had measured both $e / m$ and $e$ for the corpuscles emitted by a zinc plate when illuminated with ultra-violet light, thus identifying them with the cathode ray particles. From then onwards, as Prof. Crowther so tastefully expressed it, "the electron was accepted as a respectable member of society".

Prof. Peierls, who was introduced by Prof. G. I. Finch, president of the Physical Society, dealt with the effect which the electron, together with its associated fundamental particles, the positron, neutron, meson and neutrino, has had on the theoretical development of the laws governing the behaviour and structure of matter. The breakdown of classical mechanies led to the introduction of quantum theory and wave mechanics, with the consequent realization that the distinction between waves and corpuscles is not as fundamental as had been thought. In some respects the electron can behave as a wave, and in other respects as a corpuscle, particularly when it is moving slowly compared with the speed of light. The last remaining feature of the electron, its spin, was deduced by Uhlenbeck and Goudsmit from their work on atomic spectra. The consequences of these new laws have helped in the understanding of the structure of solids, the properties of metals and in particular the conduction of electricity through metals, leaving perhaps only one outstanding unsolved problem, superconductivity. 
J. J. Thomson himself had noted that the mass of the electron increased with the speed, the first and the simplest proof of relativity. The incorporation of the relativity idea into the equations for the electron was done brilliantly by Dirac, and this not only reproduced all the known cases for slow-moving electrons, together with a gratuitous 'spin', but also introduced negative energy states predicting the existence of the positron, discovered somewhat later. Prof. Peierls, in conclusion, referred to the presentday difficulties, stating that the electron will not be completely understood until the reason for its comparatively weak charge is known.

Sir George Thomson was welcomed by Dr. V. Z. de Ferranti, president of the Institution of Electrical Engineers, who reminded the audience that Sir George was best known for his discovery of electron diffraction, in which the electron behaves as a wave, and which has had important industrial developments.

Two applications of electrons, their use for the study of the very small, and for the production of very high voltages, were considered by Sir George, in his lecture on the electron in research. Since electrons possess wave properties analogous to light, any optical instrument has an electron analogue. The two most important are the electron microscope, which follows its optical model very closely whether employing electrostatic or magnetic lenses, and the electron diffraction camera, where the electrons are diffracted by the regular arrangement of the atoms in a crystal, similar to light by a ruled grating. Dr. Raether's work on the polishing of rock salt, in which the surface was examined both by electron diffraction and by the electron microscope, was described by Sir George, and slides shown of the results obtained to illustrate the use of electrons for the examination of the very small, and the complementary nature of the two methods.

Nuclear physics requires energies of not less than $100 \mathrm{MeV}$., so that methods of producing high voltages are becoming increasingly important. Limiting attention to electrons, the Van de Graaff electrostatic generator, the betatron, the synchrotron with its variant the microtron, and the linear accelerator are the more important machines for generating high-speed particles, and these were briefly explained by Sir George, who gave several interesting details of some of the generators now in use or under construction. The high-energy electrons, it was pointed out, are most often used to produce X-rays, of great value industrially for the examination of castings, etc., and medically for direct use in therapy.

The applications of electrons in industry was the subject of the lecture by Dr. Allibone, who followed Sir George, and who was introduced by Dr. P. Good, president-elect of the Institution of Electrical Engineers. Dr. Allibone confined his remarks to the free electrons, that is, electrons separated from the atoms to which they are usually attached. $\mathrm{He}$ pointed out that Sir J. J. Thomson in 1895 and 1897 dealt with free electrons, and that J. J. Thomson's modified Crookes tube was the forerunner of the present-day cathode ray tube, which industry has developed and is now producing at the rate of 100,000 a year, rising very shortly to a million a year. Modern developments of cathode ray tubes have concentrated on improving the electron optics, and on increasing the energy, of the electron beam, in order to meet the desire of the engineer to examine electrical transients of very short duration.
Dr. Allibone referred to several of the improvements that have been made, and to other applications of the cathode ray tube, in radar and projection television. He next reviewed thermionic emission, and the many different types of thermionic tubes, giving instances of their uses, particularly in induction and dielectric heating and in communication engineering. But J. J. Thomson's observations were not confined to electrons liberated by thermionic emission. In 1899, as Prof. Crowther mentioned earlier, he had measured $\mathrm{e} / \mathrm{m}$ for electrons emitted by photo-electric emission from a zine plate illuminated by ultraviolet light. Modern technique has now extended the photo-electric effect into the infra-red region, and the photo-electric cell finds its application in thousands of industrial processes. Dr. Allibone singled out for special mention the electron multiplier, the television camera, and the war-time development of infra-red sensitive cells. Finally, he dealt with field emission, that is, electrons made free from a surface by the action of intense external electric fields, a phenomenon which J. J. Thomson never examined himself, but which is important in several branches of vacuum engineering. Dr. Allibone concluded his interesting talk, which he had illustrated by many slides, by showing a portion of the film of Sir J. J. Thomson, which the Institution of Electrical Engineering made some ten years ago, and which represents 'J. J.' in the affectionate manner which endeared him to all.

The public lecture delivered by Sir Clifford Paterson, who has the distinction of being a past president of both the Institute of Physics and of the Institution of Electrical Engineers, was naturally less academic than the four lectures delivered before the learned societies. The audience was entertained by an instructive series of experiments and demonstrations, in which they saw how the scientific worker and the engineer, armed with the knowledge provided by J. J. Thomson that electricity consists of an immense number of minute particles, the electrons, have devised means of liberating these electrons from solids, and then developed and used every kind of free electron agency and device. Forty years of development has resulted in a vast industry, 'electronic engineering', engaged in the production of these numerous electric devices, which have changed and are indeed revolutionizing the life of the community. Two electron liberator devices in particular, the radio valve, utilizing thermionic emission from a filament and controlling the electron stream by means of the other electrodes, and the photo-electric cell, which has a specially sensitized cold surface from which electrons are liberated by the action of light, were described by Sir Clifford. To illustrate the power of the tool that the physicist has put into the hands of the electrical engineer, a most convincing demonstration was given of the conversion of sound from a gramophone record into electrical impulses, then into light waves, back into electrical and mechanical waves to sound once more, with several intermediate stages of amplification. In the latter half of the lecture, electrons in relation to lighting was discussed. The progress made in gaseous discharge tubes for illumination purposes was elegantly demonstrated, as well as the improvement in the ordinary electric lamp. It is interesting to note in this connexion that if fluorescent lamps became universal, a saving of nearly two million tons of coal a year and two large generating stations the size of that at Battersea could be effected. 
Although the celebrations were to mark the jubilee of the electron, everyone who listened to the lectures or sees the Exhibition is bound to be impressed by the still great youthfulness and vigour of the electron, for the potentialities of the new science of electronics are attracting to their study and exploitation such an amount of skill and enterprise that what has been accomplished can be regarded only as a beginning of the achievements of the liberated electron.

S. WEINTroub

\section{INTERNATIONAL STANDARDS AND UNITS OF RADIOACTIVITY}

$\mathrm{T}$ HE first international radium standard was prepared by Mme. Curie in 1911; it consisted of $21.99 \mathrm{mgm}$. of purest radium chloride. By comparing its radiation with those from other radium sources, the latter could be measured and their radium contents expressed in grams. For the measurement of radon, grams (or cubic centimetres) of this gas are impracticable as units, and it was therefore decided to use as unit of radon the quantity which is in radioactive equilibrium with $1 \mathrm{gm}$. of radium; it was called a 'curie'. Standards for radon measurements were prepared in the form of solutions of radium salts.

Since the comparison of radioactive sources with the standards always involved measurements of ionization currents, or of the number of disintegrations per unit of time, and as especially the latter figure could be determined with much higher accuracy than the weight of the radium preparation, it became customary to base the curie directly on the number of alpha-particles emitted. According to the best measurements, $1 \mathrm{gm}$. of radium and, naturally, also the radon in equilibrium with this quantity, emits per second $3 \cdot 7 \times 10^{10}$ alpha-particles; in recent practice, unknown radioactive sources were measured in curies by comparing their particle emission with this figure. Thus the curie became the unit in which the strength not only of members of the radium family, but also of any radioactive source, could be expressed.

This important change in the principle of radioactive measurements and in the definition of the curie was never officially sanctioned, because the International Radium Standard Commission which was responsible for the early conventions on radioactive standards and units has for a long time not been able to function satisfactorily. To remedy this situation, the proposal was made bv E. U. Condon and L. F. Curtiss (Rev. Sci. Instr., 17, 249; 1946) to introduce as a new unit the activity of $10^{6}$ disintegrations per second, and to call it a 'rutherford'.

It is obvious that an international agreement is imperative, whether the 'curie' in its original or in its modified form, or the new unit, the 'rutherford', should be used. The International Congress of Chemistry recently held in London offered to a number of workers in radioactivity from various countries an opportunity to discuss this question among themselves. Under the chairmanship of Prof. F. JoliotCurie (Paris), on July 19, a meeting was held by Ellen Gleditsch (Oslo), G. de Hevesy (Stockholm), Irene Joliot-Curie (Paris), C. Jacobsen (Copenhagen), W. A. Noyes, jun. (Rochester), F. A. Paneth (Durham) and G. J. Sizoo (Amsterdam) ; and after an exchange of views they decided to make the following recommendation.
A new committee on radioactive standards and units, comprising mainly representatives of the International Union of Chemistry and of the International Union of Physics, should be formed; but all the surviving members of the old International Radium Standard Commission should be invited to join them. As representatives of the International Union of Chemistry the names of E. Gleditsch, G. de Hevesy, Warren C. Johnson, F. Joliot-Curie, F. A. Paneth and G. T. Seaborg were suggested; as repre. sentatives of the International Union of Physics those of L. F. Curtiss, Sir Charles Darwin, R. B. Evans, C. Jacobsen, Irene Joliot-Curie and G. J. Sizoo.

Those present at the meeting were unanimously in. favour of preserving the name of 'curie' as the unit of radioactivity, but to define it now officially as the quantity of a radio-element which undergoes $3.700 \times$ $10^{10}$ radioactive transformations per second. The' final decision, however, will have to await the recommendations of an international committee on radioactive standards, the formation of which was advocated.

\section{QUANTITATIVE ASPECTS OF FREE RADICAL CHEMISTRY}

\author{
By Prof. H. W. MELVILLE, F.R.S. \\ University of Aberdeen
}

$\mathrm{T}$ HE Faraday Society held a two-day meoting in Oxford during September 23-25 to discuss "The Labile Molecule", or the quantitative aspects of free radical chemistry. The nature of the discussion seemed commonplace enough, and the tremendous activity in this field of chemistry is almost taken for granted. If we neglect the war years-and that is not quite justified - the subject seems to have grown up in about five scientific years, from a rather tentative qualitative notion to a subject of considerable precision requiring for its practice a wide variety of advanced techniques.

The attempts to unravel the mechanism of chemical reactions, the mechanism of which does not involve ions, is really the story of the development of the kinetics of homogeneous reactions. Many reactions, and especially those involving halogens, are known to involve free atoms as essential intermediates. These are, in fact, the activated 'molecules' in the old Arrhenius sense. But the idea that free organic radicals could function in a similar way, though possible in principle, came at a much later stage. The preparation of simple aliphatic radicals by the thermal decomposition of metallic alkyls, and later of a large number of simple organic molecules, provided the idea that radicals might play a part in the thermal decomposition of molecules the spontaneous decomposition of which was regarded as occurring simply by fission into simpler molecules but not radicals. Similarly, a great deal of work on the photochemical decomposition of compounds like the simpler aliphatic ketones could only be satisfactorily explained if it were assumed that the first step in the reaction consisted in the fission of a carbon - carbon bond with the production of two free radicals. Such experiments, however, gave little quantitative information about the reactivity of the radicals pro. duced, for the simple reason that the radicals mostly recombined, and therefore the velocity of decomposition was fixed by the initial rate of decomposition 